\title{
ON A THEOREM OF GELFAND AND KOLMOGOROFF CONCERNING MAXIMAL IDEALS IN RINGS OF CONTINUOUS FUNCTIONS
}

\section{GILLMAN, M. HENRIKSEN, AND M. JERISON}

This paper deals with a theorem of Gelfand and Kolmogoroff concerning the ring $C=C(X, R)$ of all continuous real-valued functions on a completely regular topological space $X$, and the subring $C^{*}$ $=C^{*}(X, R)$ consisting of all bounded functions in $C$. The theorem in question yields a one-one correspondence between the maximal ideals of $C$ and those of $C^{*}$; it is stated without proof in [2]. Here we supply a proof $(\$ 2)$, and we apply the theorem to three problems previously considered by Hewitt in [5].

Our first result $(\$ 3)$ consists of two simple constructions of the $Q$-space $v X$. The second ( $\$ 4)$ exhibits a one-one correspondence between the maximal ideals of $C$ and those of $C^{*}$, in a manner which may be considered qualitatively different from that expressed by Gelfand and Kolmogoroff. In our final application ( $\$ 5$ ), we confirm Hewitt's conjecture that every $m$-closed ideal of $C$ is the intersection of all the maximal ideals that contain it. In this connection, we also examine the corresponding problem for the ring $C^{*}$; we find that a necessary and sufficient condition for the theorem to hold here is that every function in $C$ be bounded.

The relevant definitions are given below.

Further applications of the Gelfand-Kolmogoroff theorem may be found in [3].

1. Introduction. We shall capitalize on known results concerning the Stone-Cech compactification $\beta X$ of $X[1 ; 6]$. This space is characterized by the following three properties: (1) $X$ is (homeomorphic with) a dense subspace of $\beta X$; (2) $\beta X$ is compact; (3) every function $f \in C^{*}$ has a (unique) continuous extension $\bar{f}$ over $\beta X$. In particular, then, the rings $C^{*}(X, R)$ and $C^{*}(\beta X, R)$ are isomorphic.

Let $f \in C$. The set $Z(f)$ of all points $p \in X$ for which $f(p)=0$ is called the zero-set of $f$. For any nonempty subset $A$ of $C$, we write $Z(A)=\{Z(f): f \in A\}$

The closure in $\beta X$ of any subset $S$ of $\beta X$ will be denoted by $\bar{S}$. In particular, the closure in $\beta X$ of a zero-set $Z(f)$ (which is a closed subset of $X)$ is denoted by $\bar{Z}(f)$.

An ideal $I$ of $C$ (or $C^{*}$ ) is called fixed if the set $\bigcap_{f \in I} Z(f)$ is non-

Presented to the Society, September 3, 1953; received by the editors July 2, 1953. 
empty; otherwise $I$ is called free. The maximal ideals of the ring $C^{*}(X, R)$ are precisely the sets

$$
M^{* p}=\left\{f: f \in C^{*}(X, R), \bar{f}(p)=0\right\} \quad(p \in \beta X)
$$

(distinct for distinct $p$ ); $M^{* p}$ is fixed or free according as $p \in X$ or $p \in \beta X-X$. Likewise, the maximal fixed ideals of $C(X, R)$ are precisely the sets

$$
M_{p}=\{f: f \in C(X, R), f(p)=0\} \quad(p \in X) .
$$

The Gelfand-Kolmogoroff theorem generalizes this assertion to the case of arbitrary maximal ideals of $C$.

\section{The Gelfand-Kolmogoroff theorem.}

Theorem 1 (Gelfand-Kolmogoroff). A subset $M$ of $C(X, R)$ is a maximal ideal of $C(X, R)$ if and only if there is a unique point $p \in \beta X$ such that $M$ coincides with the set

$$
M^{p}=\{f: f \in C(X, R), p \in \bar{Z}(f)\} .
$$

Proof. In order to prove that $M^{p}$ is a maximal ideal of $C$, it suffices, according to $[5$, Theorem 36$]$, to show that the family $Z\left(M^{p}\right)$ is a maximal subfamily of $Z(C)$ having the finite intersection property and not containing the empty set. This last condition is obviously satisfied. Now any two disjoint zero-sets are completely separated $^{1}[5$, Theorem 19], and any two completely separated subsets of $X$ have disjoint closures in $\beta X\left[1\right.$, p. 833]. It follows that $Z\left(M^{p}\right)$ has the finite intersection property. To establish maximality with respect to this property, consider any $g \in C$ for which $Z(g)$ meets every member of $\mathcal{Z}\left(M^{p}\right)$; we are to prove that $g \in M^{p}$. Let $\Omega$ be an arbitrary neighborhood (in $\beta X$ ) of $p$, and let $\Sigma$ be any neighborhood (in $\beta X$ ) of $p$ such that $\bar{\Sigma} \subset \Omega$. By normality of $\beta X$, there is an $f \in C^{*}(X, R)$ such that $\bar{f}(\bar{\Sigma})=0, \bar{f}(\beta X-\Omega)=1$. Since $X$ is dense in $\beta X$, the set $\Sigma \cap X$ is not empty, and its closure contains $p$, and we have $f(\Sigma \cap X)=0$. Hence $p \in \bar{Z}(f)$, so $f \in M^{p}$. Therefore $Z(g)$ meets $Z(f)$. Since $Z(f) \subset \Omega$, and $\Omega$ was an arbitrary neighborhood of $p$, it follows that $Z(g)$ meets every neighborhood of $p$. Consequently $p \in \bar{Z}(g)$, i.e., $g \in M^{p}$.

Conversely, let $M$ be any maximal ideal of $C(X, R)$. The family $Z(M)$ has the finite intersection property and does not contain the empty set (since $M$ is a proper ideal). Therefore, since $\beta X$ is compact, the intersection $\Delta(M)=\bigcap_{f \in M} \bar{Z}(f)$ is not empty. Clearly for each $p \in \Delta(M), M \subset M^{p}$. Since $M^{p}$ is an ideal, as has just been shown, and

1 Two sets are completely separated if there is a continuous function which is 0 on one of them and 1 on the other. 
$M$ is a maximal ideal, we have $M=M^{p}$. Uniqueness of $p$ is an immediate consequence of complete regularity of the space.

It is clear that the ideal $M^{p}$ is fixed if and only if $p \in X$, and then $M^{p}$ coincides with the set $M_{p}$ defined above.

A direct consequence of the theorem is the fact $[2 ; 5$, Theorem 46] that the space of all maximal ideals of $C$, under the Stone topology, is homeomorphic with the corresponding space of all maximal ideals of $C^{*}$, the latter constituting Stone's construction of $\beta X$ [6].

It is known that for every maximal ideal $M$ of $C$, the residue class field $C / M$ is an ordered field containing the real field $R$ [5, Theorem 41]. The maximal ideal $M$ is called real or hyper-real according as $C / M=R$ or $C / M$ contains $R$ properly. Clearly every maximal fixed ideal is real. Free ideals can be of either type.

If $M$ is hyper-real, the order in $C / M$ is non-archimedean, and there exist elements that are infinitely large and elements that are infinitely small (with respect to unity). Let $f \in C$, and denote its image in $C / M$ by $M(f)$. The order in $C / M$ is defined in such a way that $M(f)$ is infinitely large (small) if and only if, first, for every positive integer $n$ the set of all points $p \in X$ for which $f(x) \geqq n(0 \leqq f(x) \leqq 1 / n)$ is in $Z(M)$, and second, $Z(f) \notin Z(M)$.

In the ring $C^{*}$, every maximal ideal is real. Here, for every $f$, its image under the maximal ideal $M^{* p}$ is given by: $M^{* p}(f)=\bar{f}(p)$. In the ring $C$, the corresponding situation is only slightly more complicated. It is described succinctly in terms of the following notation. Let $\widehat{R}$ denote the one-point compactification of the space $R$ of reals, designating the point added to $R$ by the symbol $\infty$ (neighborhoods of $\infty$ are complements in $\widehat{R}$ of compact subsets of $R$ ). Every function $f \in C(X, R)$ may be considered as an element of the set of all continuous real-valued functions on $X$ into $\widehat{R}$, and as such it has a continuous extension $\hat{f}$ over $\beta X$ (since $\widehat{R}$ is compact; see [6, Theorem 88]). (If $f$ is bounded, then $\widehat{f}=\bar{f}$.) We have:

Theorem 2. Let $f$ be any function in the ring $C(X, R)$, and let $M^{p}$ $(p \in \beta X)$ be any maximal ideal of $C(X, R)$ (Theorem 1$)$. Then $\hat{f}(p)=\infty$ if and only if $\left|M^{p}(f)\right|$ is infinitely large, and $\widehat{f}(p)=r$ (r real) if and only if $\left|M^{p}(f)-r\right|$ is infinitely small or zero.

Proof. Suppose that $\left|M^{p}(f)\right|$ is infinitely large. Then for every positive integer $n$, the set of all $x \in X$ for which $|f(x)| \geqq n$ is in $Z\left(M^{p}\right)$. Hence $p$ is a limit point (in $\beta X$ ) of each of these sets. Consequently $\widehat{f}(p)=\infty$. The proof for the case in which $\left|M^{p}(f)-r\right|$ is infinitely small is similar. Finally, if $M^{p}(f)=r$, then $f-r \in M^{p}$, whence $p \in \bar{Z}(f-r)$, and therefore $\hat{f}(p)=r$. The converse implications follow 
from the fact that the cases listed are mutually exclusive and exhaust all possibilities (since $C / M^{p}$ is an ordered field containing the reals).

From Theorems 1 and 2 we obtain at once:

THEOREM 3. The correspondence $M^{p_{\leftrightarrow}} \leftrightarrow M^{* p}$, between the maximal ideals of $C(X, R)$ and those of $C^{*}(X, R)$, is one-one. In particular, $M^{* p}$ is the set of all $f \in C^{*}$ for which $M^{p}(f)$ is infinitely small or zero.

3. The $Q$-space $v X$. Hewitt has introduced the concept of a $Q$-space, and has shown that every completely regular space $X$ can be imbedded as a dense subspace of a $Q$-space $v X$ such that every $f \in C$ can be extended over $v X$ : the role of $v X$ with respect to the ring $C$ is analogous to that of $\beta X$ with respect to $C^{*}$. In this section we obtain two characterizations of $v X$. The first one, Theorem 4 , is [5, Theorem 58 ] stated in a somewhat different form; we include a simple proof, based upon the ideas developed in the preceding section. Our second characterization, Theorem 5 , is new.

Definition (HewitT). A completely regular space $Y$ is called a $Q$-space if every real maximal ideal of $C(Y, R)$ is fixed.

Our development of the properties of $v X$ will be based upon the following definition (which is equivalent to the one given in the proof of $[5$, Theorem 56]).

Definition. $v X$ is the set of all points $p \in \beta X$ such that for every $f \in C(X, R)$, we have $\widehat{f}(p) \neq \infty$.

This space obviously contains $X$ as a dense subspace, and every function in $C(X, R)$ can be extended continuously to the space $v X$. Thus $C(X, R)$ and $C(v X, R)$ are isomorphic, and may accordingly be identified.

TheOREM 4. Let $Y$ be a completely regular space, and let $X$ be a dense subspace of $Y$. In order that there exist a homeomorphism of $Y$ onto $v X$ leaving $X$ pointwise fixed, it is necessary and sufficient that

(1) $Y$ be a $Q$-space, and

(2) every function in $C(X, R)$ have a continuous extension over $Y$.

Proof. Necessity. It suffices to show that the space $v X$ has the stated properties. ${ }^{2}$ We have already remarked that $v X$ satisfies (2). To show that $v X$ is a $Q$-space, let $M$ be any maximal free ideal of $C(v X, R)=C(X, R)$. Then $M=M^{p}$ for some $p \in \beta X-v X$ (Theorem $1)$. By definition of $v X$, there is an $f \in C(X, R)$ for which $\widehat{f}(p)=\infty$; from Theorem 2, then, $M^{p}$ cannot be real.

Sufficiency. If $Y$ satisfies (2), then every $f \in C^{*}(X, R)$ can be ex-

2 This remark is not so trivial as it may appear; see the example below. 
tended to $\beta Y$. Also, $X$ is dense in $\beta Y$. Therefore $\beta Y$ satisfies the characteristic properties of $\beta X$, and accordingly may be identified with it. Thus we may assume that $Y \subset \beta X$. From (2) and the definition of $v X$, we infer that $C(X, R)=C(Y, R)=C(v X, R)$, and $Y C v X$. Now for every $p \in v X$, the maximal ideal $M^{p}$ of this ring is a fixed ideal of $C(v X, R)$, and is therefore real. Since $Y$ is a $Q$-space, this real ideal $M^{p}$ of $C(Y, R)$ must be fixed, i.e., $p \in Y$. Consequently, $Y \supset v X$. Therefore $Y=v X$.

It follows that if $X$ is a $Q$-space, then $v X=X$.

Observe that the condition of pointwise invariance of $X$, which appears in the statement of Theorem 4, is essential. For consider the space $X$ of all rational points of the line, and let $Y$ consist of $X$ with a single irrational point added. Then both $X$ and $Y$ are $Q$-spaces (see, e.g., [5, Theorem 53]). Hence $v X=X$. But according to a well known theorem of Cantor $[4$, p. 99, II], $Y$ is homeomorphic with $X$. Thus $X$ is a dense subspace of $Y$, which is a $Q$-space homeomorphic with $v X$. Obviously no homeomorphism of $Y$ onto $v X$ can leave $X$ invariant, and, equally clearly, condition (2) of the theorem fails.

The space $v X$ is further characterized by the property of being the smallest $Q$-space containing $X$, in the following precise sense.

TheOREM 5. Let $\left\{Y_{\lambda}\right\}$ be the family of all Q-spaces containing $X$ and contained in $\beta X$. Then their intersection $Y=\cap_{\lambda} Y_{\lambda}$ is $v X$.

We first prove two lemmas. Here it will be necessary to distinguish among ideals in rings of functions defined over different spaces. We do this by indicating the space in question, in each instance, by a subscript (e.g., the ideal $M^{p}$ of $C(X, R)$ will be denoted by $M_{X}^{p}$ ).

Lemma 1. Let $X \subset Y \subset Y^{\prime} \subset \beta X$, and let $p$ be any point of $\beta X$ $\left(=\beta Y=\beta Y^{\prime}\right)$. If the maximal ideal $M_{Y^{\prime}}^{p}$ of $C\left(Y^{\prime}, R\right)$ is hyper-real, then so is the maximal ideal $M_{Y}^{p}$ of $C(Y, R)$.

Proof. The ring $C\left(Y^{\prime}, R\right)$ may be regarded as a subring of $C(Y, R)$. Since $M_{Y^{\prime}}^{p}$ is hyper-real there is an $f \in C\left(Y^{\prime}, R\right)$ for which $M_{Y^{\prime}}^{p}(f)$ is infinitely large, i.e., $\hat{f}(p)=\infty$. Therefore $M_{Y^{\prime}}^{p}(f)$ is infinitely large, so $M_{Y}^{p}$ is hyper-real.

LEMMA 2. Let $\left\{Y_{\lambda}\right\}$ be any nonvoid family of $Q$-spaces, each containing $X$ and contained in $\beta X$. Then the intersection $Y=\cap_{\lambda} Y_{\lambda}$ is a Q-space.

Proof. Let $M_{Y}$ be any maximal free ideal of $C(Y, R)$. Then $M_{Y}=M_{Y}^{p}$ for some $p \in \beta X-Y$. Since $p \notin Y$, there is a $\lambda$ such that $p \notin Y_{\lambda}$. Then $M_{Y_{\lambda}}^{p}$ is a maximal free ideal of $C\left(Y_{\lambda}, R\right)$. Since $Y_{\lambda}$ is a 
$Q$-space, by hypothesis, this ideal is hyper-real. Hence, from the preceding lemma, the ideal $M_{Y}^{p}$ is hyper-real. It follows that $Y$ is a $Q$-space.

Proof of Theorem 5. We verify the properties (1) and (2) of Theorem 4. Note first that the family of $Q$-spaces in question is not empty, since $\beta X$ itself, being compact, is a $Q$-space. Clearly $X$ is a dense subspace of $Y$. From Lemma $2, Y$ is a $Q$-space. Finally, since $v X$ is a $Q$-space, we have $Y \subset v X$, by definition of $Y$; this implies (2).

4. Further remarks on the one-one correspondence. The correspondence $M^{p_{\leftrightarrow}} M^{*_{p}}$ of Theorem 3 is expressed in terms of the points $p$ of $\beta X$. The purpose of the theorem that follows is to provide for the passage from a given maximal ideal, of either ring, to the corresponding maximal ideal of the other, according to a rule that does not require such explicit knowledge ${ }^{3}$ of the identity of the associated point $p$ of $\beta X$. Such a passage from $C$ to $C^{*}$ has in fact already been described-in the second sentence of Theorem 3. This relation, as well as the alternate we are about to present, is also given by Hewitt, in [5, Theorem 45]. In the same theorem Hewitt describes a transition from $C^{*}$ to $C$; it has been pointed out to us by M. E. Shanks, however, that this part of the theorem is in error. ${ }^{4}$

Theorem 6. (1) For every maximal ideal $M$ of $C$, there is a unique maximal ideal $\phi(M)$ of $C^{*}$, such that $\phi(M) \supset M \cap C^{*}$; and we have $\phi\left(M^{p}\right)=M^{* p}$.

(2) For every maximal ideal $M^{*}$ of $C^{*}$, there is a unique maximal ideal $\psi\left(M^{*}\right)$ of $C$, such that $M^{*} \supset \psi\left(M^{*}\right) \cap C^{*}$; and we have $\psi\left(M^{* p}\right)$ $=M^{p}$.

Proof. (1) It is easily seen that $M^{p} \cap C^{*}$ is a prime ideal of $C^{*}$, and that $M^{p} \cap C^{*} \subset M^{* p}$. But from [3, Theorem 3.3], every prime ideal of $C^{*}$ is contained in a unique maximal ideal of $C^{*}$. This implies (1).

(2) We have already observed that $M^{* p} \supset M^{p} \cap C^{*}$. For the uniqueness, let $M^{* p} \supset M^{q} \cap C^{*}$; then $p=q$, by (1).

Hewitt has shown that the maximal ideal $M^{* p}$ of $C^{*}$ coincides with the ideal $M^{p} \cap C^{*}$ if and only if $M^{p}$ is real [5, Theorem 45].

An alternate to (2) of Theorem 6 is the statement that $M^{p}$ is the unique maximal ideal $M$ of $C$ such that $M \cap M^{* p}$ is a prime ideal of $C^{*}$. The details of the proof are easy, and may be omitted.

Theorem 7. $\left(M^{p} \cap C^{*}\right)$, the ideal of $C$ that is generated by $M^{p} \cap C^{*}$,

${ }^{3}$ Our proof, however, will make use of this knowledge.

- The family $S\left(\mathfrak{M}^{*}\right)$ need not have the finite intersection property. 
is precisely the maximal ideal $M^{p}$.

Proof. Clearly $\left(M^{p} \cap C^{*}\right) \subset M^{p}$. Conversely, let $f \in M^{p}$. Define $g(x)=1$ if $|f(x)|<1, g(x)=1 /|f(x)|$ if $|f(x)| \geqq 1$. Then both $g$ and $1 / g$ are continuous. Put $f^{*}=f g$. Then $f^{*}$ and $f$ are multiples of one another, and therefore belong to the same ideals of $C .^{5}$ Moreover, $f^{*} \in C^{*}$. Hence $f^{*} \in\left(M^{p} \cap C^{*}\right)$, and therefore $f \in\left(M^{p} \cap C^{*}\right)$.

We close this section with another simple theorem relating $C$ to $C^{*}$. First we introduce a definition and a lemma, which will also be needed later.

Definition. For every ideal $I$ of $C$ we denote by $\Delta(I)$ the set $\bigcap_{\varrho \in I} \bar{Z}(g)$. Equivalently: $\Delta(I)=\left\{p: M^{p} \supset I\right\}$.

The equivalence is a consequence of Theorem 1 .

Lemma 3. For every point $p \in \beta X-\Delta(I)$, there is an $f \in I \cap C^{*}$ such that $\bar{f}(p)=1$.

Proof. Since $p \notin \Delta(I)$, there is a $g \in I$ such that $p \notin \bar{Z}(g)$. Let $\Omega$ be a neighborhood (in $\beta X$ ) of $p$, disjoint from $\bar{Z}(g)$, and let $\Sigma$ be a neighborhood (in $\beta X$ ) of $p$ such that $\bar{\Sigma} \subset \Omega$. There is an $f \in C^{*}$ such that $\bar{f}(p)=1, \bar{f}(\beta X-\Sigma)=0$. Define $h$ according to: $h=f / g$ on $\bar{\Sigma} \cap X$, $h=0$ on $(\beta X-\bar{\Sigma}) \cap X$. Then $h \in C(X, R)$; and $f=h g$, whence $f \in I$.

THeORem 8. For every ideal $I$ of $C$ and every point $p$ of $\beta X$, we have $M^{p} \supset I$ if and only if $M^{* p} \supset I \cap C^{*}$.

Proof. Suppose $M^{p} \supset I$. Then $M^{p} \cap C^{*} \supset I \cap C^{*}$. But we have $M^{* p} \supset M^{p} \cap C^{*}$. Therefore $M^{* p} \supset I \cap C^{*}$.

Suppose $M^{p} \nsupseteq I$. Then $p \in \Delta(I)$. From the preceding lemma, there is an $f \in I \cap C^{*}$ such that $\bar{f}(p)=1$. Then $f \notin M^{* p}$. Hence $M^{* p} D I \cap C^{*}$.

5. The problem of $m$-closed ideals. Let $\Pi$ denote the family of all positive functions $\pi \in C$, i.e., such that $\pi(x)>0$ for all $x \in X$. For every $f \in C$ and $\pi \in \Pi$, let $O_{\pi}(f)$ denote the set of all $g \in C$ for which $|f-g|<\pi$, i.e., $|f(x)-g(x)|<\pi(x)$ for all $x \in X$. The topology defined on $C(X, R)$ by taking the family $\left\{O_{\pi}(f)\right\}(f \in C, \pi \in \Pi)$ as a base of open sets is called the $m$-topology (Hewitt [5, Definition 3]). $(C(X, R)$ then becomes a topological ring.) It is known that every maximal ideal of $C$ is $m$-closed [5, Theorem 39]. Here we shall verify Hewitt's conjecture that every $m$-closed ideal of $C$ is an intersection of maximal ideals.

The $m$-closure of any subset $A$ of $C$ will be denoted by $A^{m}$.

Definition. For every ideal $I$ of $C$, we denote by $K(I)$ the set of

5 It follows as a corollary that the correspondence $I \rightarrow I \cap C^{*}$ is one-one from the ideals of $C$ into the ideals of $C^{*}$. 
all $f \in C$ such that $Z(f) \supset U \supset Z(g)$ for some $g \in I$ and some open subset $U$ of $X$ for which $X-U$ is a zero-set.

The lemma that follows is due to Dr. James D. McKnight, Jr.

Lemma 4. (McKNight). For every ideal $I$ of $C$, we have $K(I) \subset I$ and $K(I)^{m}=I^{m}$.

Proof. If $f \in K(I)$, there is a $g \in I$ and an open set $U$ such that $Z(f) \supset U \supset Z(g)$. Define $h$ by: $h=0$ on $U, h=f / g$ on $X-U$. Clearly $h \in C$ and $f=g h$. Therefore $f \in I$. Hence $K(I) \subset I$.

Consider now any $g \in I$, and let $\pi \in \Pi$ be arbitrary; we shall find a function $f \in K(I)$ which lies in the $\pi$-neighborhood of $g$. Put $f(x)=0$ whenever $-\pi(x) / 2 \leqq g(x) \leqq \pi(x) / 2, \quad f(x)=g(x)-\pi(x) / 2$ if $g(x)>$ $\pi(x) / 2, f(x)=g(x)+\pi(x) / 2$ for $g(x)<-\pi(x) / 2$. Clearly $f$ is continuous, and $|g-f|<\pi$, so $f$ lies in the $\pi$-neighborhood of $g$. Let $U$ be the set of all points $x$ for which $-\pi(x) / 2<g(x)<\pi(x) / 2$. Then $U$ is open, and $Z(f) \supset U \supset Z(g)$. Moreover, $X-U=Z(h)$, where $h$ is the continuous function defined as follows: $h(x)=0$ whenever $|g(x)| \geqq \pi(x) / 2$, $h(x)=\pi(x) / 2-|g(x)|$ if $|g(x)|<\pi(x) / 2$. Therefore $f \in K(I)$. We have shown that $I \subset K(I)^{m}$. Since $K(I) \subset I$, it follows that $K(I)^{m}=I^{m}$.

Lemma 5. Let $\Omega$ be an open subset of $\beta X$ containing $\Delta(I)$. Then there exists a $g \in I \cap C^{*}$ such that $\bar{g}$ does not vanish outside $\Omega$.

Proof. For every $p \in \beta X-\Omega$, there is by Lemma 3 a $g_{p} \in I \cap C^{*}$ such that $\bar{g}_{p}(p)=1$. Let $\Sigma_{p}$ denote the set of points $q$ of $\beta X$ for which $\bar{g}_{p}(q) \neq 0$. Since $\beta X-\Omega$ is compact, it is covered by some finite union $\bigcup_{i=1}^{n} \Sigma_{p_{i}}$. Then the function $g=\sum_{i=1}^{n} g_{p_{i}}^{2}$ is as required.

THEOREM 9. The $m$-closure of any ideal $I$ of $C$ is the intersection of all the maximal ideals containing $I$.

Proof. Let $I$ be any ideal of $C$, and denote by $I^{\prime}$ the intersection of all the maximal ideals containing $I$. The ideal $I^{\prime}$ is $m$-closed, since every maximal ideal is $m$-closed. Hence it suffices to show that $K\left(I^{\prime}\right)$ $\subset K(I)$, for then, by McKnight's lemma, we shall have $I^{\prime} \subset I^{m}$.

Accordingly, let $f \in K\left(I^{\prime}\right)$; we shall prove that $f \in K(I)$. We have $Z(f) \supset U \supset Z(k)$ for some $k \in I^{\prime}$ and some open subset $U$ of $X$ such that $X-U$ is a zero-set. Put $Z=X-U$. Then $Z(k)$ and $Z$ are disjoint zero-sets; by [5, Theorem 19] there is an $h \in C^{*}$ such that $h(Z(k))=0, h(Z)=1$, and $0 \leqq h \leqq 1$ everywhere. Then $\bar{h}(\bar{Z}(k))=0$, $\bar{h}(\bar{Z})=1$. Now $\beta X=\bar{X}=\bar{U} \cup \bar{Z}$. Let $\Omega$ be the set of all points $p \in \beta X$ for which $\bar{h}(p)<1$. Then $\Omega$ is an open subset of $\beta X$, and $\bar{U} \supset \Omega \supset \bar{Z}(k)$. Now on recalling the definition of $\Delta(I)$, we observe that the relation $k \in I^{\prime}$ is equivalent to $Z(k) \supset \Delta(I)$. Therefore $\Omega \supset \Delta(I)$. From the pre- 
ceding lemma, there is a $g \in I$ such that $\Omega \supset \bar{Z}(g)$. So we have: $\bar{Z}(f)$ $\supset \bar{U} \supset \Omega \supset \bar{Z}(g)$. Intersecting these sets with $X$ yields $Z(f) \supset \Omega \cap X$ $\supset Z(g)$. Now $\Omega \cap X$ is open in $X$, and $g \in I$. Furthermore, it is clear from the definition of $\Omega$ that $X-(\Omega \cap X)$ is precisely the set $Z(1-h)$. Therefore $f \in K(I)$. This completes the proof of the theorem.

In the definition of the $m$-topology on $C$, no loss of generality results from restricting ourselves to those functions $\pi$ that are bounded. Consequently there can be no ambiguity in speaking of the $m$-topology on $C^{*}$. Note also that $C^{*}$ is an $m$-closed subring of $C$, and that every maximal ideal of $C^{*}$ is $m$-closed.

TheOREM 10. A necessary and sufficient condition that every $m$-closed ideal of $C^{*}$ be an intersection of maximal ideals of $C^{*}$ is that $C=C^{*}$ (i.e., that every $f \in C$ be bounded).

Proof. If $C=C^{*}$, then every positive function $\pi$ is bounded away from zero (since $1 / \pi$ must be bounded); hence its extension $\bar{\pi}$ is everywhere positive on $\beta X$. The theorem now follows from the corresponding theorem for the $m$-closed ideals of $C(\beta X, R)$. Conversely, if there is an unbounded $f \in C(X, R)$, then clearly there exists a hyper-real maximal ideal $M^{p}$ of $C(X, R)$ (cf. also [5, Theorem 41]). There is an $f \in C^{*}$ such that $M^{p}(f)$ is infinitely small. Hence $M^{p} \cap C^{*}$ $\neq M^{* p}$ (see also [5, Theorem 45]). It is obvious that $M^{p} \cap C^{*}$ is $m$-closed. But from (1) of Theorem $6, M^{* p}$ is the unique maximal ideal of $C^{*}$ containing $M^{p} \cap C^{*}$. The latter, therefore, is an instance of an $m$-closed ideal of $C^{*}$ that is not an intersection of maximal ideals of $C^{*}$. This completes the proof.

Added in proof. Some of the results of this paper, in particular Theorem 9, have since been obtained independently by T. Shirota; see Proc. Japan Acad., vol. 30 (1954) pp. 85-89.

\section{REFERENCES}

1. E. Čech, On bicompact spaces, Ann. of Math. vol. 38 (1937) pp. 823-844.

2. I. Gelfand and A. N. Kolmogoroff, On rings of continuous functions on topological spaces, C. R. (Doklady) Acad. Sci. URSS vol. 22 (1939) pp. 11-15.

3. L. Gillman and M. Henriksen, Concerning rings of continuous functions, Trans. Amer. Math. Soc. (to appear).

4. F. Hausdorff, Grundzüge der Mengenlehre, Leipzig, 1914.

5. E. Hewitt, Rings of real-valued continuous functions. I, Trans. Amer. Math. Soc. vol. 64 (1948) pp. 45-99.

6. M. H. Stone, Applications of the theory of Boolean rings to general topology, Trans. Amer. Math. Soc. vol. 41 (1937) pp. 375-481. 